\title{
A multi-criteria approach to robust outsourcing decision-making in stochastic manufacturing systems
}

Gerd J. Hahn ${ }^{\mathrm{a}, *}$, Torben Sens ${ }^{\mathrm{b}}$, Catherine Decouttere ${ }^{\mathrm{c}}$, Nico J. Vandaele $^{\mathrm{c}}$

${ }^{a}$ German Graduate School of Management E Law, Bildungscampus 2, 74076 Heilbronn, Germany

${ }^{b}$ Lufthansa Consulting GmbH, Hugo-Eckener-Ring 1, 60546 Frankfurt/Main, Germany ${ }^{c}$ Research Center for Operations Management, KULeuven, Naamsestraaat 69, 3000 Leuven, Belgium

\begin{abstract}
Manufacturing outsourcing is a key industry trend towards greater operations effectiveness and is related to the discussion of strategic core competencies. We study the issue of contract manufacturing at the strategic-tactical level aiming for robust decisions to accommodate stochastic manufacturing environments and immanent uncertainty of planning parameters. The topic is approached from a multi-criteria decision-making perspective, since service, cost, quality, and more long-term value-related aspects need to be considered to arrive at well-balanced decisions. Our contribution is twofold: first, we develop a scenario-based non-parametric ranking approach to determine beneficial outsourcing options at the strategic level. The ranking method uses both model-based Key Performance Indicators (KPIs), which are obtained from a tactical planning model, and non-model-based KPIs that are derived in an independent assessment from multiple stakeholders. Second, we provide an enhanced aggregate planning approach at the tactical level in order to evaluate the performance implications of the strategic outsourcing decisions which in turn serve as the modelbased KPIs for the ranking method. A queuing network-based approach is incorporated in the aggregate planning model to anticipate the stochastic behavior of manufacturing systems. An industry-derived case example with
\end{abstract}

\footnotetext{
* Corresponding author

Email addresses: gerd.hahn@ggs.de (Gerd J. Hahn), torben.sens@lhconsulting.com (Torben Sens), catherine.decouttere@kuleuven.be (Catherine Decouttere), nico.vandaele@kuleuven. be (Nico J. Vandaele)
} 
distinct outsourcing options is used to highlight the benefits of the approach and to investigate tactical trade-offs when coordinating internal and external manufacturing decisions.

Keywords: Multi-criteria decision-making, stochastic models, outsourcing, manufacturing systems, aggregate planning 
- Robust DEA-based approach for multi-criteria decision-making developed

- Partial views of DEA envelope curves provide instructive decision support

- Enhanced Aggregate planning approach for stochastic environments developed

- Demand variability drives outsourcing volumes and reduces internal batch sizes

- Higher setup variability increases insourcing volumes and average batch sizes 


\section{Introduction}

In the 1980s, a marked trend towards the reduction of internal added value was observable primarily in the automotive and service sectors (Helper, 1991; Gottfredson et al., 2005). Nowadays, in a more volatile world, corporate strategists talk about focusing on core competencies and see manufacturing outsourcing as a lever for increasing efficiency while managing operations risk. While in 1988 outsourcing in the pharmaceutical industry accounted for only around $20 \%$ of total production, in the 2000s about every 6 in 10 goods were supplied externally (van Arnum, 2000). Similarly, in 2000 outsourcing covered $10 \%$ of global electronics production (Plambeck \& Taylor, 2005). Recent data reveal that the top 25 contractors earned 342 bn USD in global sales in 2013, corresponding to some $30 \%$ of the total electronics manufacturing market (Manufacturing Market Insider, 2014) further confirms the relevance of manufacturing outsourcing.

Contract manufacturing $(\mathrm{CM})$ agreements commission the contractor to provide on-demand manufacturing of variable production volumes. In this study, we focus on turnkey CM that covers the provision of finished goods in contrast to selective outsourcing at the component level (consignment CM) (see Kim, 2003). The contractee transfers manufacturing obligations to the contractor, which increases its flexibility and can lead to cost efficiencies as well as service improvements. Moreover, operations risk with respect to quality and compliance issues can be transferred to the contractor. Despite these benefits, some fundamental strategic concerns remain. The contractee might lose critical knowhow regarding manufacturing procedures and might struggle to retain expertise in-house, while the contractor increases bargaining power and can even turn into a future competitor (Arruñada \& Vázquez, 2006). Furthermore, outsourcing entails reputational or ethical risks such as poor working standards or the violation of worker participation rights. As a consequence, manufacturing outsourcing has moved away from its purely financial focus towards a more broadly evaluated manufacturing approach. In this respect, the topic touches some fundamental lines of the business policy and thus requires comprehensive multi-dimensional decision support.

In this paper, we study the issue of selecting beneficial outsourcing options at the strategic level considering multiple financial and non- 
financial measures such as service, quality, and risk. More specifically, we use the example of turnkey $\mathrm{CM}$ as mentioned above and evaluate several contractors that could differ in manufacturing capabilities with respect to producible product range and available production capacity. When evaluating the outsourcing options, one needs to consider the impact on tactical operations performance with respect to costs and lead times since optimal capacity levels and volume/mix decisions for the internal production change according to the capabilities of the external manufacturing option. These effects are reinforced by the dynamics of stochastic manufacturing systems regarding workload-dependent lead times given the volume/mix decisions. To account for these multiple facets involving such decision-making, a decision support system needs to tackle the problem from a combined strategic-tactical perspective and must balance both qualitative and quantitative factors. While costs and lead times can be derived using quantitative models, qualitative factors such as quality or risk must be assessed by subject matter experts. Finally, a corresponding approach needs to provide robust decisions that lead to reasonable results irrespective of future developments, accommodating the strategic and partly irreversible character of outsourcing decisions.

The existing literature covers only selective aspects of the aforementioned problem, but mainly in isolation. For instance, Dotoli \& Falagario (2012) apply Data Envelopment Analysis (DEA) for supplier selection, while Merzifonluoğlu et al. (2007) consider subcontracting options in a tactical planning model and Asmundsson et al. (2009) study congestion phenomena in stochastic manufacturing systems. Moreover, robust decision-making has received increasing attention in the operations management context (see e.g., Hahn \& Kuhn, 2012). With this paper, we close a current gap in the literature by integrating strategic outsourcing decision-making with tactical operations planning to anticipate the impact of sourcing decisions on operations performance in a stochastic manufacturing environment. These inter-dependencies can have significant impact and thus influence optimal decision-making substantially as we will show below.

Our contribution lies in the integration of the strategic and the tactical planning levels while addressing the multi-dimensionality of outsourcing decision- 
making. At each point, we use state-of-the-art methodology complemented with the necessary extensions for the decision problem in focus. In our approach, we also emphasize the issue of robust decision-making to accommodate the impact of parameter uncertainty in this context. To solve the multi-criteria decision problem and to ensure robust solutions, we build on DEA to evaluate and construct the ranking of sourcing options at the strategic level given multiple scenarios. An Aggregate Production Planning (APP) model is applied at the tactical level in order to coordinate volume/mix decisions between internal and external production. In contrast to previous simulation-based approaches (see, e.g., Hung \& Leachman, 1996), we integrate a queuing network model to capture the non-linear behavior of a stochastic manufacturing system. Although we present a strategical-tactical decision framework for outsourcing decision-making, the approach is generic and can be adapted to any multi-dimensional decision problem that involves the selection of discrete design options while considering the impact on the tactical level.

The remainder of the paper is structured as follows: we review the existing literature on multi-criteria decision-making in supplier selection and aggregate production planning of stochastic manufacturing systems in section 2. In section 3, we develop an approach for robust outsourcing decision-making in a stochastic manufacturing environment. An industry-based case example is presented in section 4 to illustrate the benefits of this approach and to investigate general implications of CM decision-making on stochastic manufacturing systems. We end the paper with concluding remarks on our findings and suggest directions for future research in section 5 .

\section{Literature Review}

The relevant literature for this research follows three streams: (i) aggregate production planning with subcontracting options, (ii) production planning with an emphasis on workload-dependent lead times as well as stochastic manufacturing systems, and (iii) multi-criteria decision-making in supplier selection.

As far as the first stream is concerned, an overview of deterministic and stochastic APP approaches can be found in Nam \& Logendran (1992), Mula 
et al. (2006), and Díaz-Madroñero et al. (2014), most recently. Díaz-Madroñero et al. (2015) provide a related survey on tactical optimization models for integrated production and transportation routing problems. Building on this, Merzifonluoğlu et al. (2007) integrate subcontracting options and introduce sales price decisions as well as economies of scale using concave revenue and cost functions. Similarly, Atamtürk \& Hochbaum (2001) propose a model for balancing capacity expansion, subcontracting, internal manufacturing, and inventory holdings in a setting with non-stationary demand. The work of Carravilla \& de Sousa (1995) features subcontracting embedded in a hierarchical framework that distinguishes aggregate planning, master production scheduling, and operational loading. The issue of advanced planning and scheduling in manufacturing supply chains with an outsourcing option is considered in Lee et al. (2002). Finally, Kim (2003) proposes an optimal control model to investigate outsourcing with two contractors that provide a range of price levels and improvement capabilities for future cost savings. While these approaches incorporate subcontracting, they omit the implications of variability and assume fixed lead times irrespective of the workload in the system. The latter will be explicitly taken into account in our approach using workload-based lead times.

The second stream of literature addresses the aforementioned deficits of conventional APP models. Armbruster \& Uzsoy (2012) summarize corresponding approaches including clearing functions and discrete-event simulation (DES). An overview of clearing functions approaches that aim at capturing the nonlinear relationship between work-in-process (WIP) and the maximum output can be found in Pahl et al. (2007) and Missbauer \& Uzsoy (2011). Asmundsson et al. (2009) extend the basic clearing functions formulations of Graves (1986) and Karmarkar (1987) and develop a multi-stage multi-product planning approach. Similarly, Selçuk et al. (2008) apply clearing functions in a hierarchical supply chain planning framework to predict lead times among other operational performance metrics. However, current clearing functions-based approaches fall short of capturing the impact of lot-sizing on the performance of stochastic manufacturing systems (Missbauer \& Uzsoy, 2011).

DES represents an alternative approach to anticipate the performance of stochastic manufacturing systems in production planning which allows for a 
more detailed modeling of the shop floor level. Simulation-based approaches have received increasing attention since the early work of Hung \& Leachman (1996), which is confirmed by a series of case studies from the semiconductor industry (see, e.g., Kacar et al., 2012). Additionally, Almeder et al. (2009) outline a general framework for the iterative application of linear programming (LP) models and DES to obtain more accurate results. Gansterer et al. (2014) use simulation to improve values for lead times, safety stocks, and lot sizes in a hierarchical planning setting. However, a simulation-based optimization approach can result in prohibitive computing times (Armbruster \& Uzsoy, 2012) and thus is not in focus of this work. Moreover, the aforementioned approaches do not solve the issue of managing multiple decision criteria between the different simulated scenarios.

Multi-model approaches that combine classical APP models with more elaborate queuing models represent a compromise to resolve the aforementioned shortcomings of clearing functions-based approaches and simulation-based optimization. Corresponding early queuing-theoretical models can be found in papers of Karmarkar and co-workers (e.g., Karmarkar et al., 1985, 1992) that especially discuss the impact of batching in multi-machine multi-product job shop environments. Further contributions in this field originate from Söhner \& Schneeweiss (1995) and Lambrecht et al. (1998) that integrate queuing models into hierarchical planning frameworks for lot-sizing and scheduling. Kang et al. (2014) investigate the impact of lot-sizing in multi-product environments for single-stage systems with congestion. Lot-sizing and batching in multi-stage systems such as job shops lead to batch arrivals more downstream which need to be considered when modeling arrival rates (see section 3.2). El-Taha (2014) examine the issue of batch arrivals in queuing systems to this end.

While Jansen et al. (2013) use a local smoothing algorithm to determine aggregate order release targets and to anticipate lead times in master planning, Hahn et al. (2012) apply an aggregate stochastic queuing model to determine required capacity buffers and corresponding lead times at the tactical production planning level. None of the approaches, however, takes external capacity options and their implications on operational performance into account. The approach presented in this paper addresses these issues. 
For an overview of the third literature stream of multi-criteria decisionmaking in supplier/contractor selection, we refer the reader to the comprehensive reviews of Boer et al. (2001) and Ho et al. (2010). Dotoli \& Falagario (2012) apply a hierarchical extension of DEA in a multi-sourcing context to assess the efficiency of potential sourcing strategies. Kuo \& Lin (2012) combine DEA with the analytic hierarchy process and examine environmental protection issues in this context. Further applications of DEA in the context of product development and production planning can be found in Geng et al. (2010) and Amirteimoori \& Kordrostami (2012). However, the aforementioned approaches do not deal with the implications of sourcing decisions on operational performance of stochastic manufacturing systems and do not explicitly consider the issue of decision robustness. We close this gap by developing a multi-criteria decision support system for robust outsourcing decision-making in stochastic manufacturing systems that combines DEA with aggregate production planning. Previous work entails a similar approach that has been developed in an R\&D portfolio context by Vandaele \& Decouttere (2013).

\section{Decision Support System}

\subsection{Robust Multi-criteria Approach}

Our approach builds on the DEA methodology (see Charnes et al., 1978) in order to solve the multi-criteria decision problem of selecting beneficial outsourcing options while considering performance implications on manufacturing systems in a stochastic environment. DEA is a non-parametric approach to ranking entities with respect to their efficiency given multiple inputs and/or outputs that require a positive correlation but no formal relationship. Although inputs and outputs can have different units of measure or even represent ordinal scales, one obtains a unified comparative efficiency score. In this paper, we use an output-oriented and radial DEA model characterized by constant returns to scale. The DEA approach can be formalized as a linear program which is solved for each entity to be ranked by finding the most beneficial weights $w, v$ for the input $(x)$ and output $(u)$ factors. The mathematical model is briefly summarized in the following: 


$$
\begin{aligned}
& \max e_{0}=\sum_{j=1}^{J} w_{j} u_{i 0} \\
& \sum_{i=1}^{I} v_{i} x_{i 0}=1 \\
& \sum_{j=1}^{J} w_{j} u_{j n}-\sum_{i=1}^{I} v_{i} x_{i n} \leq 0 \\
& w_{j} \geq 0, v_{i} \geq 0 \\
& \quad j=1, \ldots, J ; i=1, \ldots, I
\end{aligned}
$$

The DEA approach maximizes the relative efficiency $e$, i.e., weighted outputs divided by weighted inputs, for the entity in focus (here denoted by 0 ). This is done by maximizing the weighted outputs in (1) while keeping the weighted inputs (normalized to 1) constant in (2). Relative efficiency cannot exceed 1 in (3) and the optimal weights are required to be non-negative in (4). With this in mind, we focus on two important facets of the aforementioned decision problem: (i) balancing multiple quantitative and qualitative factors to evaluate and rank available outsourcing options, and (ii) finding robust sourcing decisions that are sufficiently independent of uncertain future developments and accommodate the typically risk-averse preference of the decision-maker (Scholl, 2001).

First, we need to find input and output metrics in order to evaluate and rank the strategic CM options based on their DEA score. For this purpose, we rely on two sources: (i) Model-based KPIs are used to evaluate the quantitative factors of the strategic $\mathrm{CM}$ options and are derived from a tactical planning model which is capable of dealing with stochastic manufacturing environments (see subsection 3.2). (ii) Non-model-based KPIs capture the qualitative factors such as outsourcing risk or management complexity and are assessed independently by subject matter experts and/or executives using ordinal scales. Although pursuing different modeling approaches, the fundamen- 
tal architecture of our approach is similar to classical decision support systems for strategic network design in supply chain management (see Fleischmann \& Koberstein, 2015): DEA for ranking distinct CM options corresponds to the discrete strategic decision level of network design models while tactical planning models are used in both approaches to evaluate the consequences of the strategic decisions.

Second, to ensure robust decisions, we need to accommodate uncertainty concerning the input parameters. Creating a full factorial design of possible parameter constellations, we arrive at a set of scenarios which are applied to the CM options and then serve as the instances in the DEA. Having derived the DEA scores, different metrics can be used to evaluate the outsourcing options considering the risk preference of the decision-maker. While comparing minimum DEA scores between CM options corresponds to a worst-case perspective, different kinds of partial moments or related decision rules could be used to model different levels of risk aversion (see Hahn \& Kuhn, 2012). In doing this, we basically 'solve' the second stage of a two-stage stochastic problem when uncertainty has been resolved with the $\mathrm{CM}$ option being the first-stage decision (see Birge \& Louveaux, 2011). Note that probabilities for the scenarios are not required (but could be considered) and that we can easily combine multiple sources of uncertainty with even larger scenario sets.

\subsection{Aggregate Planning in Stochastic Systems}

Modeling the stochastic and dynamic behavior of a manufacturing system for planning purposes would require a stochastic non-linear programming (SNLP) approach. Building on Hahn et al. (2012), we decompose the SNLP approach into a hierarchical planning system according to Schneeweiss (2003) with a deterministic APP model as the top level and an Aggregate Stochastic Queuing (ASQ) model as the anticipated base level. This allows us to focus the tactical planning level on the key decisions (volume/mix of external vs. internal production) while capturing their non-linear performance implications. The APP model involves a linear program in order to balance capacity, WIP, and CM costs by managing volume/mix decisions given dynamic demand. Internal production volumes and capacity levels of the APP model serve as the top-down instructions for the ASQ model. 
The ASQ model covers the stochastic dimension of the problem considering demand- and supply-related variation. Workload-dependent lead times as well as capacity buffers to compensate for non-productive times are determined in the ASQ model and represent the bottom-up reaction given the instructions of the top level. Both results are incorporated as feedback in the APP model for the next iteration of the planning algorithm. Following the definition of Schneeweiss (2003), we implement perfect anticipation and thus full coordination between the two planning levels can be reached. Since we observe dynamic demand, the ASQ model needs to be solved separately for each time bucket of the APP model due to the steady state assumption. In doing this, we decompose the planning horizon into separate planning periods and apply a stationary independent period by period (SIPP) approach as introduced in Green et al. (2001) without backlog-carryover.

Both levels of the hierarchical framework are solved iteratively with the APP model also coordinating the results of the separate ASQ models. The algorithm terminates as soon as the objective value of the APP model cannot be further improved and the two planning levels are coordinated as described above. Both the APP and ASQ models as well as the iterative solution procedure are described in subsequent paragraphs using the following notation:

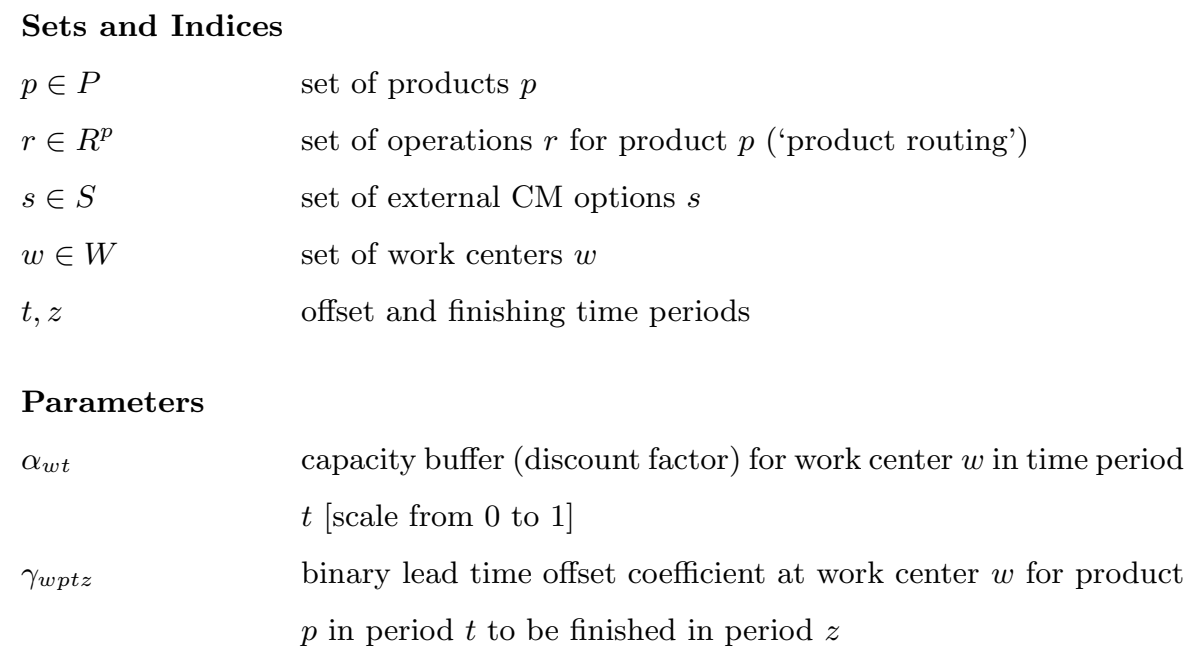




\begin{tabular}{|c|c|}
\hline$\delta_{p r w}$ & $\begin{array}{l}\text { binary parameter indicating whether operation } r \text { of product } p \\
\text { is performed at work center } w\end{array}$ \\
\hline$\lambda_{p}$ & arrival rate of orders for product $p\left(1 / \overline{y_{p}}\right)$ \\
\hline$\rho_{w}^{\prime}$ & adapted traffic intensity at work center $w$ \\
\hline$a_{w}$ & machine availability at work center $w$ \\
\hline $\operatorname{cap} X$ & maximum amount of available working time [hours] \\
\hline$c a_{w}^{2}$ & aggregate $\mathrm{SCV}$ of the batch interarrival time at work center $w$ \\
\hline$c s_{w}^{2}$ & aggregate SCV of the batch processing time at work center $w$ \\
\hline$c m_{p s}$ & unit cost of CM for product $p$ of contractor $s$ \\
\hline co & cost of overtime [monetary unit/hour] \\
\hline$c w_{p}$ & unit cost of holding WIP for product $p$ \\
\hline$d_{p t}$ & demand for product $p$ in period $t$ \\
\hline$k x_{w p}$ & capacity requirement at work center $w$ for product $p$ [hours/unit] \\
\hline$l_{w}$ & aggregate batch arrival rate at work center $w$ \\
\hline$l t_{p t}$ & lead time for product $p$ in period $t$ [periods] \\
\hline$M_{p s t}^{\max }$ & $\begin{array}{l}\text { maximum allowable } \mathrm{CM} \text { amount of product } p \text { in period } t \text { by } \\
\text { contractor } s\end{array}$ \\
\hline$n m_{w}$ & number of parallel machines at work center $w$ \\
\hline$O^{\max }$ & maximum allowable overtime usage [hours] \\
\hline$\overline{o q_{p}}$ & average order size of product $p$ \\
\hline$\overline{p t_{p r}}$ & average processing time of operation $r$ of product $p$ \\
\hline$\overline{s t_{p r}}$ & average setup time of operation $r$ of product $p$ \\
\hline$w q_{w}$ & average waiting time in queue in front of work center $w$ \\
\hline$\overline{y_{p}}$ & average interarrival time for orders of product $p$ \\
\hline
\end{tabular}

\section{Decision variables}

$\begin{array}{ll}M_{p s t} & \text { external production quantity of product } p \text { in period } t \text { by con- } \\ & \text { tractor } s \\ O_{t} & \text { amount of overtime used in time period } t \\ Q_{p} & \text { batch size of product } p \text { (number of orders in one batch) } \\ X_{p t} & \text { internal production quantity of product } p \text { in period } t\end{array}$

APP Model. As a starting point for the multi-period APP model, we assume a multi-product make-to-order job shop setting where overtime can be used to adapt to changing demand levels and backlogs are not permitted. We incorporate a form of CM that can be used up to a certain allowable volume to serve customer demand at a fixed unit cost. The 
second group of enhancements centers around workload-dependent lead times and necessary capacity buffers that are derived from the ASQ models in order to anticipate the impact of volume/mix decisions in the APP model. Lead times are considered in the APP model in two ways: first, lead times are translated into average WIP costs which need to be balanced against capacity and CM costs. Second, lead times are used to allocate capacity requirements to the respective lead time offset periods and work centers given the product routing and due date. The capacity buffer corresponds to a discount factor on gross capacity at the APP level to account for non-productive time due to setups and machine breakdowns.

Consequently, the objective function in (5) minimizes total relevant costs consisting of three elements: (i) the costs of overtime derived from the overtime quantity $O_{t}$ in period $t$ and the corresponding unit cost $c o$, (ii) the costs of CM derived from the external production quantity $M_{p s t}$ per product $p$ and sourcing option $s$ involving the respective unit cost $c m_{p s}$, and (iii) the holding costs of WIP inventory per product $p$ until the planning horizon $T$. WIP holding costs per product $p$ and period $t$ are derived from the production lead time $l t_{p t}$ and the production amount $X_{p t}$ using average WIP cost $c w_{p}$ per time bucket. The lead time coefficient $l t_{p t}$ captures the average lead time (expressed in time buckets) and is determined in the ASQ model as described further below. In doing this, we assume that WIP holding costs are linear in production volumes and depend on average lead times invoking Little's law.

$$
\min \sum_{t=1}^{T}\left(c o \cdot O_{t}\right)+\sum_{p \in P} \sum_{s \in S} \sum_{t=1}^{T}\left(c m_{p s} \cdot M_{p s t}\right)+\sum_{p \in P} \sum_{t=1}^{T}\left(c w_{p} \cdot l t_{p t} \cdot X_{p t}\right)
$$

The capacity restriction in (6) is modified in two ways: on the RHS, the capacity buffer $\alpha_{w t}$ represents a discount on the gross capacity given the number of parallel machines $n m_{w}$, the standard working time $\operatorname{cap} X$, and the amount of overtime $O_{t}$ in period $t$. On the LHS, the capacity load per product $p$ is derived from the production amount $X_{p z}$ and the capacity requirement $k x_{w p}$ at work center $w$. The binary coefficient $\gamma_{w p t z}$ determines whether a certain 
operation of product $p$ at work center $w$ is assigned to a particular lead time offset period $t\left(\gamma_{w p t z}=1\right)$ with the end item to be finished in period $z$. In doing this, we consider multi-stage product routings which typically occur in job shop settings and involve significant lead times that can be spread across multiple periods. Both the capacity buffer $\alpha_{w t}$ and the lead time offset parameter $\gamma_{w p t z}$ are workload-dependent and thus are derived from the ASQ models as described below.

$$
\begin{aligned}
& \sum_{p \in P} \sum_{z=t}^{T} \gamma_{w p t z} \cdot k x_{w p} \cdot X_{p z} \leq\left(1-\alpha_{w t}\right) \cdot n m_{w} \cdot\left(c a p X+O_{t}\right) \\
& \forall w \in W ; t=1 \ldots T
\end{aligned}
$$

Equation (7) represents the mass balance constraint for a make-to-order environment. Consequently, demand $d_{p t}$ for product $p$ in period $t$ must be satisfied with items either produced internally $\left(X_{p t}\right)$ or sourced externally from a contractor $\left(M_{p s t}\right)$. In accordance with the hierarchical approach as described above, we treat demand as a deterministic parameter in the APP model and consider the implications of demand variability in the ASQ models. Maximum overtime $O^{\max }$ per period $t$ is defined in (8). Equation (9) ensures that agreed-to CM volumes $M_{p s t}^{\max }$ for product $p$ and sourcing option $s$ are not exceeded.

$$
\begin{array}{lr}
X_{p t}+M_{p s t}=d_{p t} & \forall p \in P ; \forall s \in S ; t=1 \ldots T \\
O_{t} \leq O^{\max } & t=1 \ldots T \\
M_{p s t} \leq M_{p s t}^{\max } & \forall p \in P ; \forall s \in S ; t=1 \ldots T \\
& \\
M_{p s t}, X_{p t}, O_{t} \geq 0 & \forall p \in P ; \forall s \in S ; t=1 \ldots T
\end{array}
$$

$$
\forall p \in P ; \forall s \in S ; t=1 \ldots \cdot T
$$

Lastly, all decision variables need to be restricted to the non-negative domain 
in (10). Solving the APP model using a standard LP solver, one obtains optimal internal and external production quantities per product and period as well as the necessary overtime capacities.

ASQ Model. The ASQ model considers a multi-machine job shop and is derived using queuing network approximations as presented in Lambrecht et al. (1998). The model determines lead time-optimal batch sizes $Q_{p}$ for each product $p$ assuming a strict first-come, first-served discipline and no batch splitting along the product routing. Since the approach considers average order sizes $\overline{o q_{p}}$, the decision variable $Q_{p}$ is a multiplier and counts the number of orders that are grouped together in one batch. Consequently, the number of units processed in one batch equals the product of the average order size $\overline{o q_{p}}$ and the batch size $Q_{p}$ for each product. The binary parameter $\delta_{p r w}$ indicates whether operation $r$ of product $p$ is performed on a specific work center $w$ and thus contains the product routing information.

The objective function of the ASQ model in (11) covers the weighted average lead time that is aggregated over all products of the job shop. The weighting factor $\lambda_{p} \cdot \overline{o q_{p}} / \sum_{p \in P} \lambda_{p} \cdot \overline{o q_{p}}$ represents the relative importance of product $p$ for the overall job shop according to the interarrival rate $\lambda_{p}$ of product $p$ compared with the sum of interarrival rates of all products. The lead time consists of the waiting time to batch in front of the first work center, the waiting time in the queue at each work center, and the setup and processing times at each working center along the product routing. The first term of (11) captures the waiting time to batch in front of the first work center for given product-specific average interarrival times $\overline{y_{p}}$.

$$
\begin{gathered}
\min _{Q_{p}} \sum_{p \in P} \frac{\lambda_{p} \cdot \overline{o q_{p}}}{\sum_{p \in P} \lambda_{p} \cdot \overline{o q_{p}}} \cdot \frac{\left(Q_{p} \cdot \overline{o q_{p}}-1\right) \cdot \overline{y_{p}}}{2 \cdot \overline{o q_{p}}}+\sum_{w \in W} E\left(w q_{w}\right) \\
+\sum_{w \in W} \sum_{p \in P} \frac{\sum_{r \in R^{p}} \lambda_{p} \cdot \overline{o q_{p}} \cdot \delta_{p r w}}{\sum_{p \in P} \sum_{r \in R^{p}} \lambda_{p} \cdot \overline{o q_{p}} \cdot \delta_{p r w}} \cdot \\
\quad\left(\sum_{r \in R^{p}} \frac{\lambda_{p} \cdot \overline{o q_{p}} \cdot \delta_{p r w}}{\sum_{r \in R^{p}} \lambda_{p} \cdot \overline{o q_{p}} \cdot \delta_{p r w}} \cdot\left(\overline{s t_{p r}}+Q_{p} \cdot \overline{o q_{p}} \cdot \overline{p t_{p r}}\right)\right)
\end{gathered}
$$

$E\left(w q_{w}\right)$ denotes the expected waiting time in the queue at each work center $w$ and is derived in (12) using a modified version of the well-known Kraemer 
and Langenbach-Belz approximation (see Kraemer \& Langenbach-Belz, 1976). Equation (12) uses aggregates of the batch arrival rates and the aggregate SCVs of the batch interarrival and processing times at work center $w$ that consider the product routings of the orders. $l_{w}$ denotes the aggregate batch arrival rate at work center $w$ which equals $\sum_{p \in P} \sum_{r \in R^{p}} \lambda_{p} / Q_{p} \cdot \delta_{p r w}$. The batch interarrival and processing times at work center $w, c a_{w}^{2}$ and $c s_{w}^{2}$, are determined according to Lambrecht et al. (1998).

$$
E\left(w q_{w}\right)= \begin{cases}\frac{\rho_{w}^{\prime 2} \cdot\left(c a_{w}^{2}+c s_{w}^{2}\right)}{2 \cdot l_{w} \cdot\left(1-\rho_{w}^{\prime}\right)} \cdot \exp \left\{\frac{-2 \cdot\left(1-\rho_{w}^{\prime}\right) \cdot\left(1-c a_{w}^{2}\right)^{2}}{3 \cdot \rho_{w}^{\prime} \cdot\left(c a_{w}^{2}+c s_{w}^{2}\right)}\right\} & \text { if } c a_{w}^{2} \leq 1 \\ \frac{\rho_{w}^{\prime} \cdot\left(c a_{w}^{2}+c s_{w}^{2}\right)}{2 \cdot l_{w} \cdot\left(1-\rho_{w}^{\prime}\right)} & \text { if } c a_{w}^{2}>1\end{cases}
$$

The basic assumption is that for all operations the aggregate batch arrival rate equals the aggregate batch departure rate $\left(1-\rho_{w}^{\prime 2}\right) \cdot c a_{w}^{2}+\rho_{w}^{\prime 2} \cdot c s_{w}^{2}$ of previous operations at work center $w$. This represents a convex combination of the aggregates of the interarrival time SCV and the processing time SCV. If traffic intensity is low, the SCV of the aggregate batch departure time is close to the aggregate interarrival time SCV; otherwise, the SCV is approximately equal to the aggregate processing time SCV under high capacity usage. In (13), $\rho_{w}^{\prime}$ represents the adapted traffic intensity for work center $w$ that accounts for the additional utilization due to setups.

$$
\rho_{w}^{\prime}=\sum_{p \in P} \sum_{r \in R^{p}} \frac{\lambda_{p}}{Q_{p}} \cdot \delta_{p r w} \cdot \overline{s t_{p r}}+\rho_{w} \quad \forall w \in W
$$

The last term of the objective function in (11) calculates expected weighted setup and processing times. Setup times $\overline{s t_{p r}}$ and processing times $\overline{p t_{p r}}$ for operation $r$ of product $p$ also follow general distributions that are characterized by their means and SCVs. Average setup and processing times as well as their corresponding standard deviations need to be divided by the availability $a_{w}$ of work center $w$ to obtain effective values (see Hopp \& Spearman, 2011). It should be noted that this represents only a rough approximation which is found to be appropriate in this case (for further details see Vandaele et al. (2003)). The model has been adapted to integrate parallel servers and to account for the impact of machine breakdowns. The corresponding revisions are described 
in detail in Lieckens \& Vandaele (2012). The ASQ model is solved using a dedicated optimization routine as described in Lambrecht et al. (1998).

Iterative Solution Procedure. The iterative solution procedure follows three steps: first, the APP model is solved with initial assumptions on the lead times ( $l t_{p t}$ and $\left.\gamma_{w p t z}\right)$ and the capacity buffer $\alpha_{w}$ that can be derived from long-run empirical observations. Second, one ASQ model per period $t$ of the APP model is solved using interarrival times $\overline{y_{p t}}$ for orders of product $p$ which are derived in (14) from internal production volumes of the APP model and corresponding working times. Consequently, we manage workload in the ASQ models via the arrival stream of orders into the manufacturing system.

$$
\overline{y_{p t}}=\frac{c a p X+O_{t}}{X_{p t}} \quad t=1 \ldots T
$$

Third, we use the results of the ASQ models to update lead time as well as capacity buffer parameters (see step 1) and then re-run the APP model. For this purpose, the aggregate lead time $l t_{p t}$ can be directly derived from the ASQ models considering working time in the respective time bucket of the APP model. Assuming latest possible start dates of batches to serve customer demand, we can update the binary lead time offset coefficients $\gamma_{w p t z}$ using the aggregate lead time $l t_{p t}$. More precisely, we start in time bucket $z$ when the batch needs to be finished and iterate backwards on the operations that belong to the product routing of the respective product $p$. For each operation, the algorithm checks whether the partial lead time of the operation fits into the current time bucket $t$. If so, there is a capacity requirement in period $t$ on work center $w$ for the respective product $p$ according to the product routing and thus $\gamma_{w p t z}$ is set equal to one. Otherwise, the offset period $t$ is increased by one and the algorithm re-checks feasibility of the partial lead time in the respective time bucket.

The required capacity buffer $\alpha_{w}$ for work center $w$ is derived using machine downtime and total setup time at the respective work center in period $t$. Machine availability is given as a fixed parameter $a_{w}$, while total setup time needs to be calculated from the setup times of the respective operations at the work center and the required number of setups given the production volume in the 


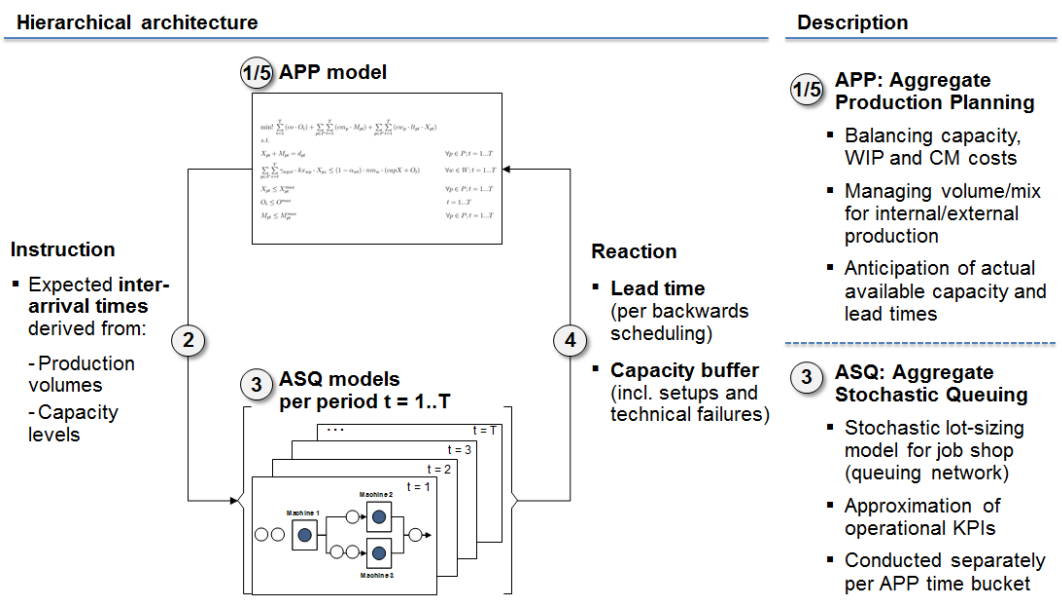

Figure 1: Architecture of the aggregate planning approach

respective lead time offset period as well as the batch size of the product. Note that the capacity buffer in terms of idle time (to cope with small variations in the system parameters) has been taken into account when solving the ASQ model. Steps 2 and 3 of the iterative solution procedure are repeated until the two planning levels are coordinated, i.e., the algorithm terminates as soon as the relative deviation of two consecutive objective function values of the APP model falls below a given threshold value.

To ensure coordination of the two planning levels, we gradually reduce the interval between minimum and maximum batch sizes in the ASQ models relative to the optimal batch sizes from the previous iteration. Consequently, the solution space reduces and the change in the objective function value for consecutive iterations decreases. Eventually, minimum and maximum batch sizes will coincide and the optimal solution of the APP model remains unchanged with the algorithm terminating at latest at this point. The relationship of the the APP and ASQ models and the iterative solution procedure are visualized in Figure 1.

\subsection{Solution Procedure}

Having outlined the building blocks of our approach in the previous sections, we describe the solution procedure step-by-step in the following: 
I. Define design options: The set of possible design options needs to be defined from which the most beneficial one should be selected. In our case, a design option corresponds to a CM option which could be one contractor or a group of contractors with different manufacturing capabilities.

II. Create scenarios: Given uncertain parameters, we describe the different discrete scenarios that could be observed. Assuming multiple sources of uncertainty such as different levels of demand seasonality or demand variability, we need to create a full factorial design to obtain the complete set of scenarios. For example, one scenario could be the event of high seasonality while demand variability is low.

III. Assess design options: Each design option needs to be evaluated for each of the scenarios in order to obtain the model-based and non-model-based KPIs as described above. For this purpose, the aggregate planning approach is applied to each scenario-design option combination (called instance in the sequel) in order to determine the model-based KPIs. The non-model-based KPIs are derived by subject matter experts using ordinal scales.

IV. Rank design options: Mapping the relevant KPIs of the previous step to input and output factors, the DEA is conducted and aggregate measures of the DEA scores are derived per design option reflecting the risk preference of the decision-maker. In the most simplest way of a risk-neutral preference of the decision maker, one could calculate the average of the DEA scores per design option and create the ranking upon this measure to find the most beneficial outsourcing option.

4. Numerical Study

\subsection{Problem Setting and Approach}

For the numerical analysis we build on the case study of a medium-sized European automotive supplier which is described in Vandaele et al. (2000). The firm is affiliated with a large multi-national corporation and serves a range of 
customers in the B2B market. Our data set is based on a job shop environment with twelve products and ten machines. Manufacturing is performed during one shift of eight hours per day five days a week, yielding a total of 160 hours per month. This capacity can be extended to 280 hours using overtime for 500 monetary units $(\mathrm{mu})$ per hour. The WIP cost factor is set to $3 \mathrm{mu}$ per item and period. The planning horizon is divided into six periods with a length of two months each to span a full seasonal cycle.

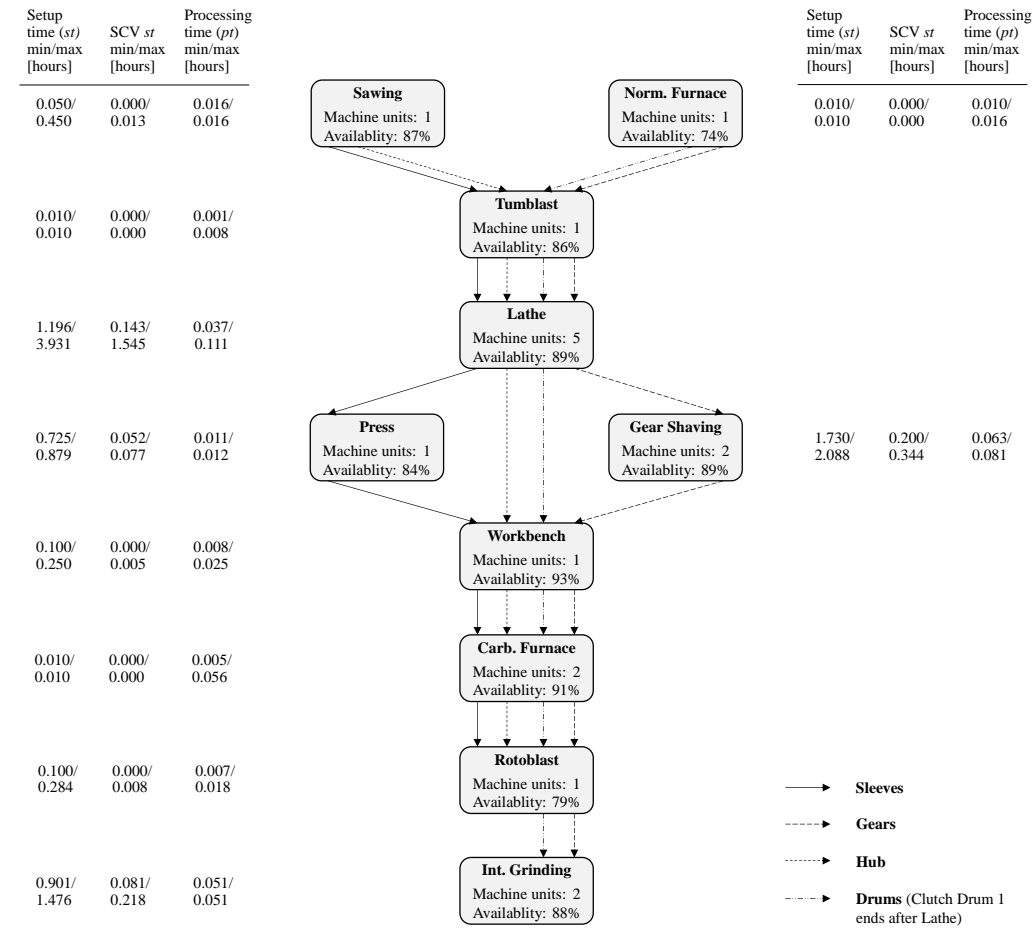

Figure 2: Product routings, machine data, and setup and processing times

The product portfolio consists of four product families: sleeves, gears, drums, and hubs. Their production processes can be summarized in four steps. After the raw steel is processed through rough machining operations in the cold steel shop, a heat-treatment hardens the parts. These hardened components are in turn treated by machining operations in the hard steel shop. In a last step, the rotating steel parts are installed into the housings and the products are finally assembled. Our case focuses on the first production phase which is the raw steel department. Figure 2 summarizes the master data pertaining to the machines 
and product routings as well as the ranges of setup and processing times. The processing times are deterministic while the setup times exhibit a certain degree of variability due to the complexity of the manual setup activities. The ranges of corresponding SCVs of the setup times are also given in Figure 2.

\begin{tabular}{cc}
\hline \hline Product name & Base demand \\
\hline Clutch Drum 1 & 95 \\
Clutch Drum 2 & 594 \\
Drum 1 & 1,320 \\
Drum 2 & 679 \\
Gear 54 Teeth & 651 \\
Gear 65 Teeth & 849 \\
Gear 69 Teeth & 54 \\
Hub & 458 \\
Sleeve 1 & 189 \\
Sleeve 2 & 500 \\
Sleeve 3 & 349 \\
Sleeve 4 & 236 \\
\hline
\end{tabular}

Table 1: Product master data

Demand follows a seasonal pattern with the base demand per product as shown in Table 1. Modeled as a harmonic oscillation, the demand peaks in the middle of the planning period and has a maximal amplitude according to a defined seasonality factor. Since the company in focus makes-to-order as to serve its own customers' assembly schedules, final goods inventories are nonexistent. Every demand must be satisfied in the respective period and cannot be delayed beyond the due date. Besides the seasonal pattern, the arrivals of customer orders can exhibit variability which we investigate further below.

Having described the key parameters and assumptions of the case study, we follow the 4-step approach described in subsection 3.3.

I. Define design options: The firm can source from two contractors that differ in terms of flexibility and price. Flexibility in this context relates to the range of products and the maximum volumes that can be commissioned to the contractor. Price levels are reflected in the unit cost of CM. Consequently, we consider three $\mathrm{CM}$ options: single sourcing from an inflexible or flexible contractor and a mixed setting with dual sourcing. The flexible contractor is capable of providing $35 \%$ of the base demand of every product for a given unit price between 3 and $15 \mathrm{mu}$. The inflexible contractor is more limited and can only deliver $20 \%$ of the base demand of the drums and hubs, albeit at a price 
discount of $35 \%$ compared with the flexible contractor.

II. Create scenarios: We consider three sources of uncertainty affecting the planning parameters: setup time variability, variability (of seasonally adjusted) demand, and demand seasonality. For the setup time variability, we distinguish three states (low, moderate, high) with respect to the product- and operation-specific SCVs. The case of low variability is characterized by the base data given in Figure 2. We apply a linear transformation to the values of the base case in order to consider moderate and high variability of setup times. Demand variability (adjusted for seasonality) originates from volatile customer order behavior and is captured in product-specific SCVs for which we also investigate three distinct cases: deterministic demand as well as moderate and high demand variability. Distinct demand seasonality factors complete the parameter constellations in focus and cover flat versus fluctuating demand over the planning period. The parameter constellations are summarized in Table 2 . Creating a full factorial design, we obtain 18 distinct scenarios.

\begin{tabular}{|c|c|c|}
\hline \multicolumn{3}{|c|}{ Parameter constellations } \\
\hline Setup variability & $\begin{array}{l}\text { low } \\
\text { moderate } \\
\text { high }\end{array}$ & $\begin{array}{l}\text { values given in the base case } \\
\text { constant term of } 0.3 \text { and scaling factor of } 1.2 \\
\text { constant term of } 0.8 \text { and scaling factor of } 2.0\end{array}$ \\
\hline Demand variability & $\begin{array}{l}\text { deterministic } \\
\text { moderate } \\
\text { high }\end{array}$ & $\begin{array}{l}S C V \text { of demand }=0.0 \\
S C V \text { of demand }=1.0 \\
S C V \text { of demand }=2.5\end{array}$ \\
\hline Demand seasonality & $\begin{array}{c}\text { flat } \\
\text { fluctuating }\end{array}$ & $\begin{array}{l}\text { seasonality factor }=10 \% \\
\text { seasonality factor }=35 \%\end{array}$ \\
\hline
\end{tabular}

Table 2: Parameter constellations for the scenario analysis

\section{Assess design options: Combining three CM options with 18}

scenarios, we obtain 54 instances for which we need to conduct the aggregate planning approach and an independent assessment of the qualitative factors. Aggregate planning is performed using IBM ILOG OPL with CPLEX v12.6.0.0 for the APP model and a steepest descent algorithm implemented in $\mathrm{C}++$ for the ASQ model. The iterative solution procedure was run on an Intel Core i5 machine with $2.7 \mathrm{GHz}$ per core and $8 \mathrm{~GB}$ RAM, resulting in computation times of around three minutes per scenario. While the execution of the APP and 
ASQ models requires only a few seconds, the data consolidation and transformation between the calculation steps accounts for most of the computation time. The iterative algorithm terminates after two to five iterations when applying a threshold value of $0.5 \%$ on the relative deviation in the objective function of APP model between two consecutive iterations. As explained above, we ensure coordination by reducing the interval between minimum and maximum batch sizes in the ASQ models over the course of five iterations. From the aggregate planning models, we obtain CM volumes, aggregate order lead times, and total relevant costs. CM volumes represent the total number of production units that are sourced from a contractor over the course of the planning period. Aggregate order lead times are derived as weighted averages across planning periods and products according to the internal production volumes. Total relevant costs are calculated as the sum of overtime, CM, and WIP costs over the planning period and can be extracted from the objective function of the APP model.

For this case, we apply a single metric to capture outsourcing risk as the major qualitative factor using an ordinal scale from 1 (low risk) to 10 (high risk). In practice, these values are assessed through structured interviews and consensus group decision-making with relevant stakeholders. In a consensusseeking workshop, decision-making guidelines are agreed upon which can be used to implement risk assessment decision rules. This is both confirmed by our industrial experience and by others in the literature. See for instance Sheikh et al. (2015) where also a systematic risk assessment approach is suggested for alternative energy system planning. For the purpose of this paper, we compare and systematically rank scenarios according to the risk content building on the observation that a scenario with an inflexible contractor as well as high demand seasonality and high demand and setup variability exhibits the highest risk. In contrast, the lowest risk level is observed when a flexible contractor is opted for in a setting with flat seasonality, deterministic demand, and low setup time variability. More formally, based on a full factorial design, we obtain the following set of instances:

1. CM option: flexible, mixed (inflexible+flexible), inflexible

2. Demand seasonality: flat, fluctuating

3. Demand variability: deterministic, moderate, and high 
4. Setup variability: low, moderate, high

The risk content is decreasing from factor 1 (CM option) to factor 4 (setup variability) and increasing within each factor from left to right according to the risk impact. Consequently, we obtain a ranked order of the instances from 1 through 54 in terms of overall risk which is rescaled afterwards to a scale from 1 to 10. As a result, we obtain an individual risk assessment for each of the 54 instances. The aggregated risk view across all scenarios per CM option is considered as a robust approach being coherent with the risk preferences of the decision-makers.

IV. Rank design options: Our DEA approach combines two inputs (risk, costs) and two outputs (internal aggregate order lead times, external CM quantities) which are all measured in categorically distinct units of measure: percentage, monetary units, time and countable units. Since lead times are fixed with the contractor irrespective of the outsourcing volume, internal lead times are a key output factor to keep under control and the CM quantities are considered to support the strategic considerations that a higher outsourced volume is preferable, provided reasonable costs and operational effects. The latter is taken care of in the APP-ASQ model. Given the general idea of DEA, we examine sourcing decisions for which risk and costs are minimized and for which external $\mathrm{CM}$ volumes and the inverse of aggregate internal lead times are maximized. The lead time metric needs to be inverted to ensure a positive correlation between input and output factors according to the fundamental assumptions of the DEA approach. The DEA efficiency scores are derived using SAS/OR 13.1 at negligible run times.

Detailed results of steps III and IV are provided in Table 4 and are further discussed in the following subsection.

\subsection{Discussion of the Results}

In the following, we concentrate on interpreting the results pertaining to the exemplary selection of dominant - or at least partially beneficial - CM options and the general implications of such a selection on operations performance.

Selection of Beneficial CM Options. The overall results are summarized in Table 5 along the three sourcing options, showing minimum, average, and 


\begin{tabular}{|c|c|c|c|c|c|c|c|c|c|}
\hline \multirow[b]{3}{*}{$\#$} & \multirow{3}{*}{$\begin{array}{c}\text { Designs } \\
\text { Sourcing } \\
\text { option }\end{array}$} & \multirow{2}{*}{\multicolumn{3}{|c|}{ Parameters }} & \multicolumn{3}{|c|}{ Model KPIs } & \multirow{3}{*}{$\begin{array}{c}\text { Non-Model } \\
\text { KPIs } \\
\text { Risk } \\
\text { [value] }\end{array}$} & \multirow{3}{*}{$\begin{array}{l}\text { DEA } \\
\text { Score }\end{array}$} \\
\hline & & & & & \multicolumn{2}{|c|}{ APP } & ASQ & & \\
\hline & & $\begin{array}{c}\text { Demand } \\
\text { seasonality }\end{array}$ & $\begin{array}{c}\text { Demand } \\
\text { variability }\end{array}$ & $\begin{array}{c}\text { Setup } \\
\text { variability }\end{array}$ & $\begin{array}{l}\text { Cost } \\
{[\mathrm{mu}]}\end{array}$ & $\begin{array}{c}\mathrm{CM} \\
{[\text { units] }}\end{array}$ & $\begin{array}{c}\mathrm{LT} \\
{[\text { hours] }}\end{array}$ & & \\
\hline 1 & flex & flat & det. & low & 63,162 & 694 & 70.0 & 1 & 80.1 \\
\hline 2 & flex & flat & det. & mod. & 57,537 & 1,467 & 56.1 & 1 & 100.0 \\
\hline 3 & flex & flat & det. & high & 68,700 & 583 & 77.9 & 1 & 72.0 \\
\hline 4 & flex & flat & mod. & low & 63,353 & 690 & 70.2 & 2 & 67.9 \\
\hline 5 & flex & flat & mod. & mod. & 57,593 & 1,453 & 56.2 & 2 & 92.1 \\
\hline 6 & flex & flat & mod. & high & 68,874 & 580 & 78.1 & 2 & 56.8 \\
\hline 7 & flex & flat & high & low & 63,657 & 685 & 70.7 & 2 & 67.2 \\
\hline 8 & flex & flat & high & mod. & 57,690 & 1,435 & 56.5 & 2 & 91.6 \\
\hline 9 & flex & flat & high & high & 58,047 & 2,143 & 52.1 & 2 & 100.0 \\
\hline 10 & flex & fluct. & det. & low & 82,755 & 4,240 & 51.2 & 3 & 100.0 \\
\hline 11 & flex & fluct. & det. & mod. & 86,659 & 3,737 & 61.6 & 3 & 88.0 \\
\hline 12 & flex & fluct. & det. & high & 89,604 & 3,596 & 66.7 & 3 & 84.6 \\
\hline 13 & flex & fluct. & mod. & low & 85,479 & 3,803 & 59.4 & 3 & 89.6 \\
\hline 14 & flex & fluct. & mod. & mod. & 86,815 & 3,729 & 61.9 & 3 & 87.8 \\
\hline 15 & flex & fluct. & mod. & high & 89,746 & 3,588 & 67.0 & 3 & 84.4 \\
\hline 16 & flex & fluct. & high & low & 85,715 & 3,790 & 59.8 & 4 & 81.5 \\
\hline 17 & flex & fluct. & high & mod. & 87,059 & 3,717 & 62.3 & 4 & 79.0 \\
\hline 18 & flex & fluct. & high & high & 89,957 & 3,579 & 67.4 & 4 & 74.2 \\
\hline 19 & mixed & flat & det. & low & 52,549 & 2,493 & 48.1 & 4 & 100.0 \\
\hline 20 & mixed & flat & det. & mod. & 61,670 & 733 & 71.0 & 4 & 60.2 \\
\hline 21 & mixed & flat & det. & high & 65,464 & 628 & 76.2 & 4 & 53.6 \\
\hline 22 & mixed & flat & mod. & low & 52,593 & 2,478 & 48.2 & 5 & 99.6 \\
\hline 23 & mixed & flat & mod. & mod. & 61,872 & 726 & 71.3 & 5 & 57.2 \\
\hline 24 & mixed & flat & mod. & high & 65,623 & 625 & 76.4 & 5 & 50.4 \\
\hline 25 & mixed & flat & high & low & 60,378 & 785 & 69.2 & 5 & 60.4 \\
\hline 26 & mixed & flat & high & mod. & 62,171 & 715 & 71.7 & 5 & 56.6 \\
\hline 27 & mixed & flat & high & high & 65,871 & 620 & 76.7 & 5 & 50.0 \\
\hline 28 & mixed & fluct. & det. & low & 77,361 & 3,860 & 60.9 & 6 & 78.9 \\
\hline 29 & mixed & fluct. & det. & mod. & 72,941 & 4,713 & 47.0 & 6 & 100.0 \\
\hline 30 & mixed & fluct. & det. & high & 81,900 & 3,580 & 68.8 & 6 & 70.5 \\
\hline 31 & mixed & fluct. & mod. & low & 77,522 & 3,850 & 61.2 & 6 & 78.6 \\
\hline 32 & mixed & fluct. & mod. & mod. & 72,990 & 4,702 & 47.2 & 6 & 99.7 \\
\hline 33 & mixed & fluct. & mod. & high & 82,032 & 3,571 & 69.0 & 6 & 70.3 \\
\hline 34 & mixed & fluct. & high & low & 77,780 & 3,835 & 61.7 & 7 & 76.3 \\
\hline 35 & mixed & fluct. & high & mod. & 73,069 & 4,688 & 47.5 & 7 & 99.3 \\
\hline 36 & mixed & fluct. & high & high & 82,224 & 3,559 & 69.4 & 7 & 67.0 \\
\hline 37 & inflex & flat & det. & low & 57,050 & 1,007 & 64.0 & 7 & 69.2 \\
\hline 38 & inflex & flat & det. & mod. & 58,770 & 897 & 66.7 & 7 & 64.4 \\
\hline 39 & inflex & flat & det. & high & 62,969 & 720 & 72.7 & 7 & 55.2 \\
\hline 40 & inflex & flat & mod. & low & 57,240 & 990 & 64.4 & 8 & 68.6 \\
\hline 41 & inflex & flat & mod. & mod. & 59,015 & 884 & 67.1 & 8 & 63.8 \\
\hline 42 & inflex & flat & mod. & high & 63,222 & 711 & 73.1 & 8 & 54.7 \\
\hline 43 & inflex & flat & high & low & 57,553 & 969 & 64.9 & 8 & 67.6 \\
\hline 44 & inflex & flat & high & mod. & 59,368 & 868 & 67.6 & 8 & 62.9 \\
\hline 45 & inflex & flat & high & high & 63,592 & 698 & 73.6 & 8 & 54.0 \\
\hline 46 & inflex & fluct. & det. & low & 82,002 & 1,371 & 55.7 & 9 & 55.3 \\
\hline 47 & inflex & fluct. & det. & mod. & 81,007 & 1,545 & 50.5 & 9 & 61.7 \\
\hline 48 & inflex & fluct. & det. & high & 85,886 & 1,299 & 64.2 & 9 & 45.8 \\
\hline 49 & inflex & fluct. & mod. & low & 82,127 & 1,364 & 56.0 & 9 & 54.9 \\
\hline 50 & inflex & fluct. & mod. & mod. & 81,064 & 1,535 & 50.8 & 9 & 61.3 \\
\hline 51 & inflex & fluct. & mod. & high & 86,062 & 1,296 & 64.6 & 9 & 45.5 \\
\hline 52 & inflex & fluct. & high & low & 82,340 & 1,358 & 56.5 & 10 & 54.3 \\
\hline 53 & inflex & fluct. & high & mod. & 81,151 & 1,524 & 51.2 & 10 & 60.8 \\
\hline 54 & inflex & fluct. & high & high & 86,310 & 1,293 & 65.0 & 10 & 45.0 \\
\hline
\end{tabular}

Table 4: Results of the DEA for the 54 scenarios 
maximum DEA scores across the scenarios. Recall that overall efficiency scores of the DEA range between 0 and 1. A single sourcing agreement with the inflexible contractor yields a mean value of only 0.58 while single sourcing with the flexible contractor and dual sourcing result in considerably higher mean DEA scores of 0.83 and 0.74 , respectively. Comparing the ranges of the DEA values, we observe a relatively narrow range of outcomes between 0.45 and 0.69 for the inflexible contractor option in contrast to significantly wider ranges for the flexible contractor option (0.57-1.00) and dual sourcing (0.50-1.00). We conclude that the flexible contractor option as well as dual sourcing largely outperform outsourcing to the inflexible contractor. Taking into account the variation of the scores, we observe that the flexible option exhibits less variation and thus is more robust given parameter uncertainty.

\begin{tabular}{c|ccc}
\hline \hline \multirow{2}{*}{$\begin{array}{c}\text { Sourcing } \\
\text { Option }\end{array}$} & \multicolumn{3}{|c}{ DEA Scores } \\
& Minimum & Average & Maximum \\
\hline flexible & 0.57 & 0.83 & 1.00 \\
mixed & 0.50 & 0.74 & 1.00 \\
inflexible & 0.45 & 0.58 & 0.69 \\
\hline
\end{tabular}

Table 5: Minimum, average, and maximum DEA scores

Continuing the investigation of the DEA results, we create partial views in terms of envelope curves along the input and output dimensions. Since we have four variables, it is impossible to have a planar representation of this fourdimensional ranking problem. However, complementing the summary statistics in Table 5, the partial views allow us to highlight additional characteristics of the instances while keeping visualization complexity at a reasonable level. Since the partial views shown in Figures 3 and 4 cover one output factor and two input factors at a time, we need to consider the overall DEA scores of the instances to interpret the results. In total, we obtain five efficient instances from the DEA which are marked with circles: instances 2, 9, and 10 are scenarios of the flexible contractor option while instances 19 and 29 belong to the mixed sourcing option. Note that only scenario 2 is shown on the envelope curve in both partial views. Both partial views confirm that scenarios with the inflexible sourcing option are generally inferior to those involving the flexible contractor arrangement with the scenarios involving the mixed setting falling in between. 
Analyzing the results for the output dimension CM volume in relation to the two input dimensions risk and costs, respectively, one can see on the vertical axis that higher risk penalizes the inflexible contractor option more than the mixedsource arrangement, which in turn is mostly inferior to all scenarios involving the flexible contractor option. We conclude that the higher risk is not compensated for by higher contractor output. Reasoning horizontally, the relative cost level with respect to $\mathrm{CM}$ volumes to costs clearly discriminates between the setting with an inflexible contractor and the other two sourcing options. However, the cost dimension does not discriminate between the flexible and the mixed sourcing option. As there are some efficient scenarios below the envelope curve of this partial view, we conclude that inverse lead time (which is not depicted here) obviously has an additional impact on the ranking of the instances.

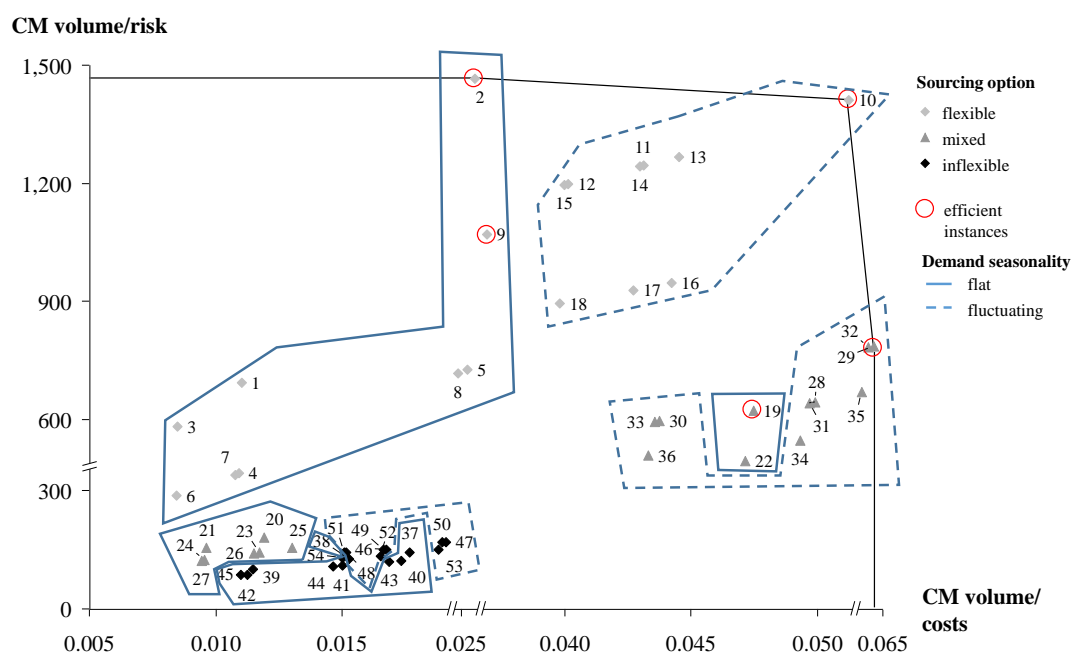

Figure 3: Envelope curve relating CM volumes to risk and costs

Comparing scenario clusters in Figure 3 (see solid and dashed clusters), one can see the impact of demand seasonality on the ranking of distinct sourcing options. While we observe a clear separation for the flexible contractor option (instances 1-9 vs. 10-18) and the mixed-source arrangement (instances 19-27 vs. 28-36) except for instances 19 and 22, there is no clear analogous separation for the scenarios involving the inflexible contractor option. This can be explained by the small product range and limited production volumes that can be commissioned to the inflexible contractor. Thus, the company cannot 
leverage the inflexible contractor to fully accommodate seasonal demand fluctuation which results in lower relative DEA factor values. Even in a mixed-source setting with additional benefits from lower CM costs, the negative impact of inflexibility remains. Examining the aforementioned scenario clusters in further detail, we observe more scattered patterns for the flexible contractor option which reconfirms the flexibility to also adapt for lower levels of demand and setup variability resulting in lower relative costs levels.

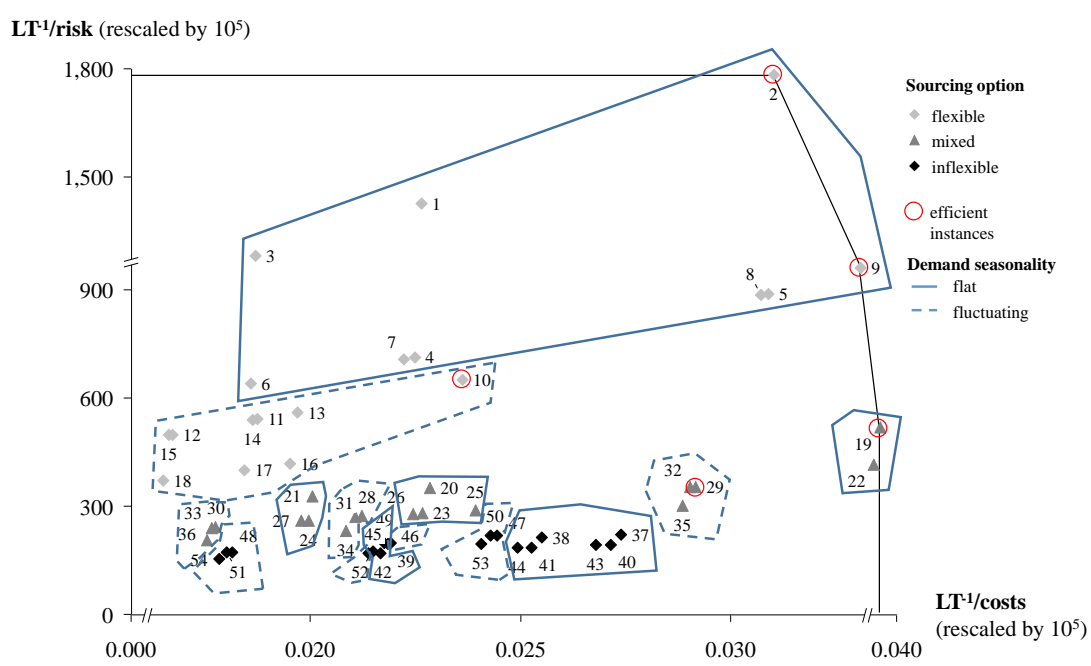

Figure 4: Envelope curve relating aggregate order lead times (LT) to risk and costs

Examining the partial view of the inverse lead time dimension (see Figure 4), we see opposite effects concerning demand seasonality such that scenarios under fluctuating demand (instances 10-18, 28-36, and 46-54) result in relatively higher risk and costs levels compared to scenarios under flat demand. This is especially true for the scenarios with higher setup variability (instances 12, 15,18 and 30,33, 36 and 48, 51,54). In general, we see a clear separation of the sourcing options with respect to the relative risk exposure, i.e., the flexible contractor option entails the lowest relative risk while the inflexible contractor options exhibits the highest risk. In contrast to the flexible contractor option and the dual sourcing setting, the results of the scenarios for the inflexible contractor option are located at the same relative risk level and only differ with respect to the relative costs level. Although the inflexible contractor options represents the least expensive outsourcing option in absolute terms, we observe 
scenarios in the flexible contractor and mixed-source agreement which realize lower relative costs levels compared to the inflexible contractor option. These scenarios largely coincide with the efficient instances from the DEA.

Summarizing the results, we clearly see three benefits of the proposed robust multi-criteria approach. First, the ranking-based methodology provides comprehensible and decisive results that can be easily interpreted and communicated especially to practitioners. This relates to both the DEA scores and the partial views. In particular, the partial views help to identify relevant factors and to assess their impact on the KPIs. In our case, the analysis revealed that the assessment of the inflexible option is mainly dominated by the risk factor while the other two sourcing options depend on both the cost and risk factors. Second, multiple qualitative and quantitative factors with different unit of measures can be incorporated into the decision process which is especially relevant for strategic decision-making since further aspects beyond costs play a more dominant role at this level. Multi-criteria optimization approaches have higher formal requirements with respect to modeling and data preparation and thus typically result in higher efforts. Third, introducing scenarios allows us to consider the impact of parameter uncertainty in order to arrive at more robust results. From our viewpoint, the presented approach is advantageous for one-off decisions with a manageable set of discrete design options as for the case of contractor selection decisions.

Operations Implications of Contract Manufacturing. In the following, we examine the stochastic manufacturing system in greater detail to investigate the impact of demand and setup variability as well as demand seasonality under two contract manufacturing options (inflexible vs. flexible). Starting with demand seasonality, we compare the cost split of total relevant costs as well as average aggregate batch sizes for flat and fluctuating demand (see Figure 5) assuming moderate demand and setup variability. We observe significantly higher relevant costs $(+37-51 \%)$ associated when demand fluctuates. Higher flexibility of the contractor implies rising $\mathrm{CM}$ costs: $+15 \mathrm{pp}$ and $+31 \mathrm{pp}$ for flat and fluctuating demand, respectively. For the case of fluctuating demand, we furthermore find 

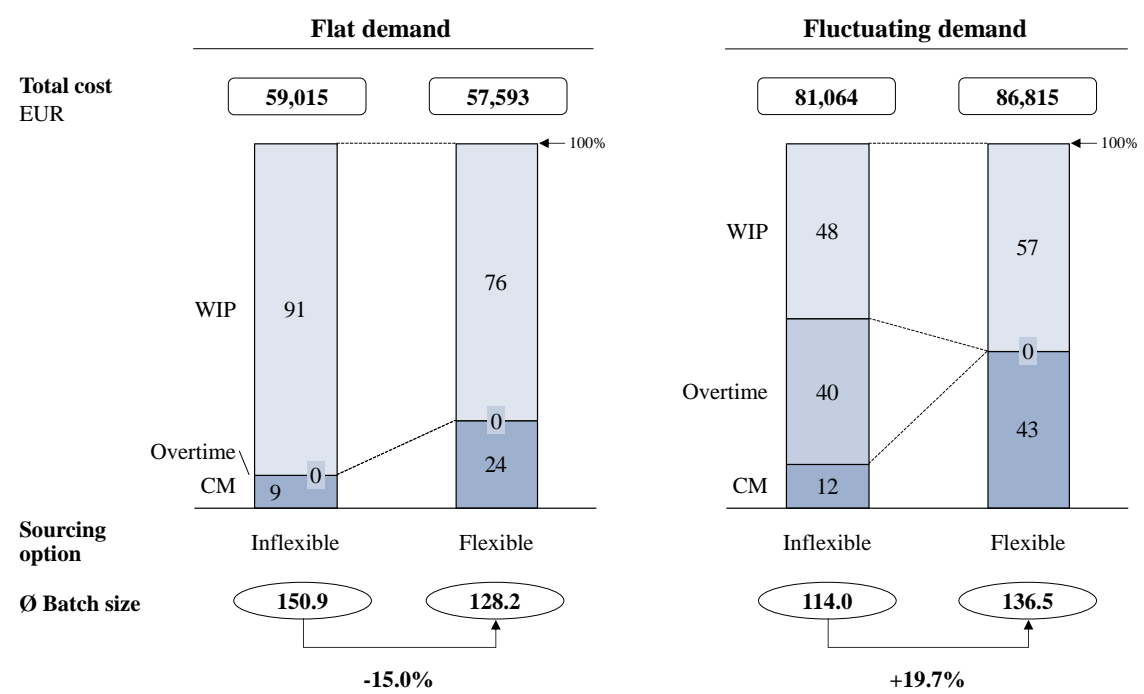

Figure 5: Aggregate costs and batch sizes for flat vs. fluctuating demand

that overtime for internal production is eliminated when switching from the inflexible to the flexible sourcing option. The corresponding effects on operations performance are reflected in average batch sizes with $-15.0 \%$ for flat demand but $+19.7 \%$ for fluctuating demand. Consequently, with flat demand, CM is used for selective outsourcing to streamline internal production by lowered average batch sizes. In contrast, when demand seasonality is high, flexible external production can serve as a buffer (analogous to overtime) in order to absorb the fluctuation. In our case, the flexible contractor option cannot fully replace internal overtime production and thus average batch sizes increase. By this means, CM in a make-to-order setting plays a similar role as finished goods inventories usually do in an MTS setting by smoothing operating levels.

As a second step, we examine the impact of demand variability for flat and fluctuating demand separately assuming moderate setup variability. From Figure 6 one can see that demand variability basically has no significant impact on outsourcing volumes and average batch sizes. Changes in the cost split are in a range of $1 \mathrm{pp}$ and average batch sizes slightly increase by $0.5-1.1 \%$ while overall cost levels remain basically constant. Reinforcing the previous results, we find that batch sizes are decreasing in outsourcing flexibility for flat demand but increasing for fluctuating demand. 


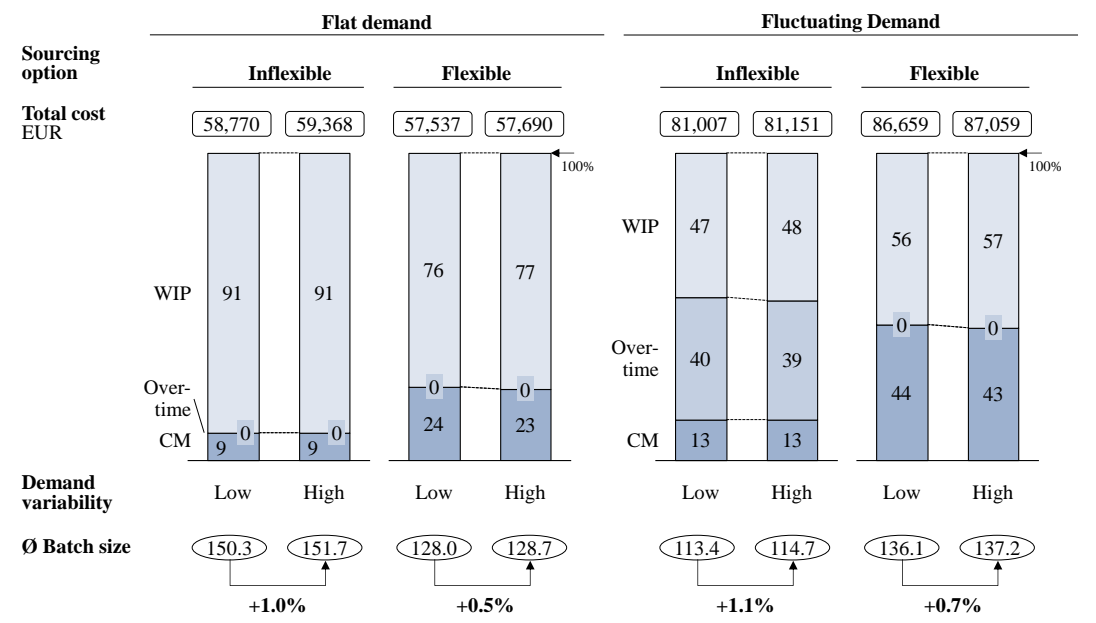

Figure 6: Aggregate costs and batch sizes for low vs. high demand variability (flat vs. fluctuating demand)

Lastly, we analyze the impact of setup variability on operations performance for flat and fluctuating demand assuming moderate demand variability (see Figure 7). Increasing setup variability leads to higher average batch sizes in a range of $+7.9-12.0 \%$ and higher costs in a range of $+4.8-10.5 \%$. At the same time, we observe a shift towards internal production and the reduction of overtime usage. This can be explained by the fact that the negative impact of setup variability is accommodated by making less frequent but larger batches. Consequently, production volumes are insourced in order to fill up the batches. Moreover, internal overtime usage is reduced to avoid costly loss of capacity due to more variable setup times. It is interesting to see that changes in demand and supply variability have different implications on outsourcing volumes and average internal batch sizes irrespective of the flexibility of the contractor: to avoid dilutions due to demand fluctuations, we focus on the case of flat demand and find that additional demand variability leads to additional outsourcing and lower internal batch sizes while increasing setup variability induces opposite results.

Summarizing the results, we clearly observe the benefits of the proposed aggregate planning approach for stochastic environments which allows us to evaluate different outsourcing options concerning their aggregate performance in costs, lead times, and average batch 


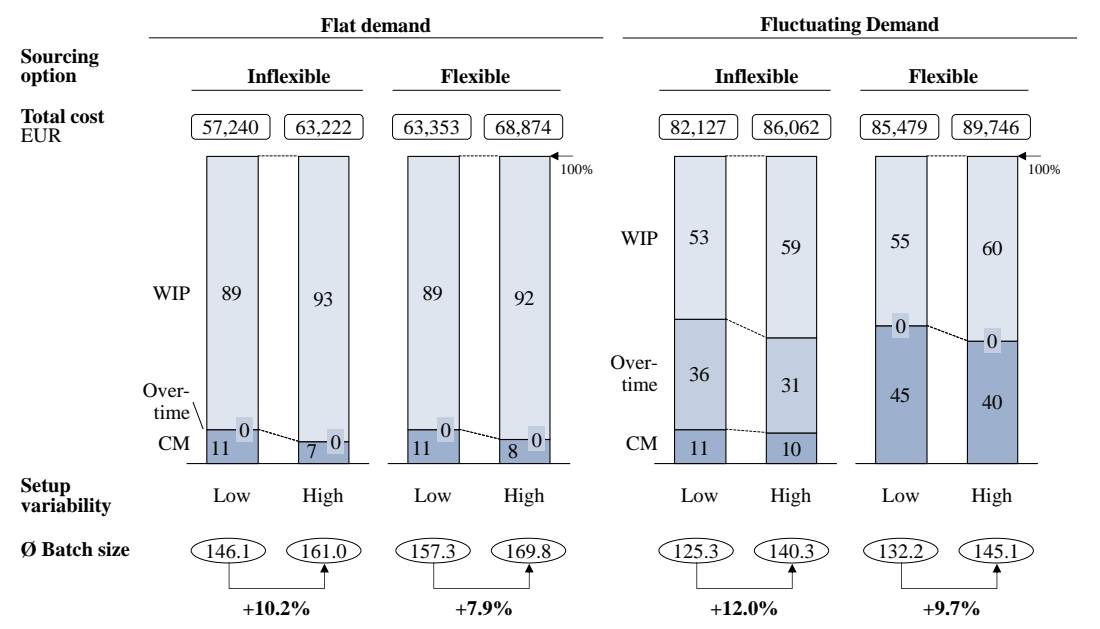

Figure 7: Aggregate costs and batch sizes for low vs. high setup variability (flat vs. fluctuating demand)

sizes. Conventional approaches typically omit the impact of system variability and use exogenously determined fixed lead times and batch sizes irrespective of the workload in the system. When assessing the combined APP and ASQ approach, it needs to be considered that a hierarchical decomposition is applied to tackle the stochastic nonlinear decision problem.

In order to quantify the benefits of the proposed approach, we use the 54 instances from the previous analysis and calculate total relevant costs when applying a conventional APP approach. For this purpose, we implicitly assume fixed lead times of one time bucket (as it is done in conventional APP models) and apply a capacity buffer for each work center of $25 \%$ which approximately corresponds to the average capacity buffer across all work centers and instances in the combined APP and ASQ approach. Comparing the results, we find an improvement potential of $51 \%$ to $64 \%$ in total relevant costs for the combined APP and ASQ approach. The substantial savings mainly result from improved internal capacity utilization and corresponding reductions in costly external production volumes. 


\section{Conclusion and Outlook}

In this paper, we proposed a novel approach to address the strategic-tactical issue of outsourcing decision-making in a stochastic manufacturing environment. Our contribution is twofold: first, we presented a robust multi-criteria approach that considers multiple quantitative as well as qualitative factors and that accounts for parameter uncertainty. For this purpose, a DEA-based approach was applied to rank several outsourcing options under various uncertain parameter constellations at the strategic level. Second, we introduced an APP approach for stochastic manufacturing environments with outsourcing options to obtain tactical performance metrics for the DEA. To this end, an aggregate stochastic queuing model was integrated to capture workload-dependent lead times as well as capacity losses due to setup times and machine availability. In summary, we provide an alternative approach to solving a multi-criteria two-stage robust design problem for a manufacturing system that is subject to variability.

A real-life case example was used to highlight our approach in which costs and risk exposure of the manufacturing system in focus were balanced against contractor output and customer service, which we represented by aggregate order lead times. For the analysis, we used a combination of overall DEA scores and partial views of envelope curves to ease representation of the results given the multi-dimensional approach of DEA. These methods appeared to be very instructive and could support executive meetings with management professionals that have only limited knowledge of OR methods. The numerical study revealed some general insights with respect to capacity management and outsourcing in stochastic manufacturing environments. Demand fluctuation appears to be a dominant factor that influences system performance and determines the strategic role of contract manufacturing especially in make-to-order settings by smoothing operating levels. However, on a tactical level, we find that variability has different implications for the management of manufacturing outsourcing: while increasing demand variability drives outsourcing volumes and reduces internal batch sizes, higher setup variability leads to opposite effects.

For future research, one can generalize the approach to include other types 
of decisions that are at stake in the S\&OP context. This could include, for instance, aspects of new product introduction and profitable-to-promise planning. In this work, we integrated outsourcing risk as the single most relevant qualitative factor. However, there are many additional intangible criteria such as sustainability, strategic fit, or managerial complexity that could be considered to this end. Furthermore, this approach could be applied to a process industry context to elaborate on the impact of variable processing times and more complex changeover processes as well as product routings. Some additional work could be done from a methodological perspective by improving the aggregate stochastic queuing model with respect to batch splitting or the modeling of machine repair activities. Moreover, the robust DEA approach should be investigated further to examine novel methods for scenario analysis as well as for visualizing and interpreting the results.

\section{References}

Almeder, C., Preusser, M., \& Hartl, R. F. (2009). Simulation and optimization of supply chains: Alternative or complementary approaches? OR Spectrum, 31, 95-119.

Amirteimoori, A., \& Kordrostami, S. (2012). Production planning in data envelopment analysis. International Journal of Production Economics, 140, $212-218$

Armbruster, D., \& Uzsoy, R. (2012). Continuous dynamic models, clearing functions, and discrete-event simulation in aggregate production planning. In P. B. Mirchandani (Ed.), TutORials in Operations Research: New directions in informatics, optimization, logistics, and production (pp. 103-126). Hanover: INFORMS volume 9.

Arruñada, B., \& Vázquez, X. H. (2006). When your contract manufacturer becomes your competitor. Harvard Business Review, 84, 135-144.

Asmundsson, J., Rardin, R. L., Turkseven, C. H., \& Uzsoy, R. (2009). Production planning with resources subject to congestion. Naval Research Logistics, $56,142-157$. 
Atamtürk, A., \& Hochbaum, D. S. (2001). Capacity acquisition, subcontracting, and lot sizing. Management Science, 47, 1081-1100.

Birge, J. R., \& Louveaux, F. (2011). Introduction to Stochastic Programming. New York: Springer.

Boer, L. d., Labro, E., \& Morlacchi, P. (2001). A review of methods supporting supplier selection. European Journal of Purchasing \& Supply Management, 7, 75-89.

Carravilla, M. A., \& de Sousa, J. P. (1995). Hierarchical production planning in a make-to-order company: A case study. European Journal of Operational Research, 86, 43-56.

Charnes, A., Cooper, W. W., \& Rhodes, E. (1978). Measuring the efficiency of decision making units. European Journal of Operational Research, 2, 429-444.

Díaz-Madroñero, M., Mula, J., \& Peidro, D. (2014). A review of discrete-time optimization models for tactical production planning. International Journal of Production Research, 52, 5171-5205.

Díaz-Madroñero, M., Peidro, D., \& Mula, J. (2015). A review of tactical optimization models for integrated production and transport routing planning decisions. Computers 83 Industrial Engineering, .

Dotoli, M., \& Falagario, M. (2012). A hierarchical model for optimal supplier selection in multiple sourcing contexts. International Journal of Production Research, 50, 2953-2967.

El-Taha, M. (2014). Sample-path analysis of queues with batch arrivals. Computers $\mathcal{E}$ Industrial Engineering, 70, 98-106.

Fleischmann, B., \& Koberstein, A. (2015). Strategic network design. In H. Stadtler, C. Kilger, \& H. Meyr (Eds.), Supply Chain Management and Advanced Planning (pp. 107-123). Berlin: Springer.

Gansterer, M., Almeder, C., \& Hartl, R. F. (2014). Simulation-based optimization methods for setting production planning parameters. International Journal of Production Economics, 151, 206-213. 
Geng, X., Chu, X., Xue, D., \& Zhang, Z. (2010). An integrated approach for rating engineering characteristics' final importance in product-service system development. Computers \& Industrial Engineering, 59, 585-594.

Gottfredson, M., Puryear, R., \& Phillips, S. (2005). Strategic sourcing: From periphery to the core. Harvard Business Review, 83, 132-139.

Graves, S. C. (1986). A tactical planning model for a job shop. Operations Research, 34, 522-533.

Green, L. V., Kolesar, P. J., \& Soares, J. (2001). Improving the SIPP approach for staffing service systems that have cyclic demands. Operations Research, $49,549-564$.

Hahn, G. J., Kaiser, C., Kuhn, H., Perdu, L., \& Vandaele, N. J. (2012). Enhancing aggregate production planning with an integrated stochastic queuing model. In D. Klatte, H.-J. Lüthi, \& K. Schmedders (Eds.), Operations Research Proceedings 2011 Operations Research Proceedings (pp. 451-456). Berlin: Springer.

Hahn, G. J., \& Kuhn, H. (2012). Value-based performance and risk management in supply chains: A robust optimization approach. International Journal of Production Economics, 139, 135-144.

Helper, S. (1991). Strategy and irreversibility in supplier relations: The case of the U.S. automobile industry. The Business History Review, 65, 781-824.

Ho, W., Xu, X., \& Dey, P. K. (2010). Multi-criteria decision making approaches for supplier evaluation and selection: A literature review. European Journal of Operational Research, 202, 16-24.

Hopp, W. J., \& Spearman, M. L. (2011). Factory physics. New York: McGrawHill/Irwin.

Hung, Y.-F., \& Leachman, R. C. (1996). A production planning methodology for semiconductor manufacturing based on iterative simulation and linear programming calculations. IEEE Transactions on Semiconductor Manufacturing, 9, 257-269. 
Jansen, M. M., de Kok, T. G., \& Fransoo, J. C. (2013). Lead time anticipation in supply chain operations planning. OR Spectrum, 35, 251-290.

Kacar, N. B., Irdem, D. F., \& Uzsoy, R. (2012). An experimental comparison of production planning using clearing functions and iterative linear programming-simulation algorithms. IEEE Transactions on Semiconductor Manufacturing, 25, 104-117.

Kang, Y., Albey, E., Hwang, S., \& Uzsoy, R. (2014). The impact of lot-sizing in multiple product environments with congestion. Journal of Manufacturing Systems, 33, 436-444.

Karmarkar, U. S. (1987). Lot sizes, lead times and in-process inventories. Management Science, 33, 409-418.

Karmarkar, U. S., Kekre, S., \& Kekre, S. (1985). Lotsizing in multi-item multimachine job shops. IIE Transactions, 17, 290-298.

Karmarkar, U. S., Kekre, S., \& Kekre, S. (1992). Multi-item batching heuristics for minimization of queueing delays. European Journal of Operational Research, 58, 99-111.

Kim, B. (2003). Dynamic outsourcing to contract manufacturers with different capabilities of reducing the supply cost. International Journal of Production Economics, 86, 63-80.

Kraemer, W., \& Langenbach-Belz, M. (1976). Approximate formulae for the delay in the queueing system $G I / G / 1$. International Teletraffic Congress.

Kuo, R. J., \& Lin, Y. J. (2012). Supplier selection using analytic network process and data envelopment analysis. International Journal of Production Research, 50, 2852-2863.

Lambrecht, M. R., Ivens, P. L., \& Vandaele, N. J. (1998). Aclips: A capacity and lead time integrated procedure for scheduling. Management Science, 44 , $1548-1561$.

Lee, Y. H., Jeong, C. S., \& Moon, C. (2002). Advanced planning and scheduling with outsourcing in manufacturing supply chain. Computers $\&$ Industrial Engineering, 43, 351-374. 
Lieckens, K., \& Vandaele, N. J. (2012). Multi-level reverse logistics network design under uncertainty. International Journal of Production Research, 50, $23-40$.

Manufacturing Market Insider (2014). MMI articles. URL: http://mfgmkt.com/mmi-articles.html. Last accessed: 06/28/2014.

Merzifonluoğlu, Y., Geunes, J., \& Romeijn, H. E. (2007). Integrated capacity, demand, and production planning with subcontracting and overtime options. Naval Research Logistics, 54, 433-447.

Missbauer, H., \& Uzsoy, R. (2011). Optimization models of production planning problems. In K. G. Kempf, P. Keskinocak, \& R. Uzsoy (Eds.), Planning Production and Inventories in the Extended Enterprise (pp. 437-507). Boston: Springer.

Mula, J., Poler, R., García-Sabater, J. P., \& Lario, F. C. (2006). Models for production planning under uncertainty: A review. International Journal of Production Economics, 103, 271-285.

Nam, S.-J., \& Logendran, R. (1992). Aggregate production planning: A survey of models and methodologies. European Journal of Operational Research, 61 , $255-272$.

Pahl, J., Voß, S., \& Woodruff, D. L. (2007). Production planning with load dependent lead times: An update of research. Annals of Operations Research, 153, 297-345.

Plambeck, E. L., \& Taylor, T. A. (2005). Sell the plant? The impact of contract manufacturing on innovation, capacity, and profitability. Management Science, 51, 133-150.

Schneeweiss, C. (2003). Distributed decision making: A unified approach. European Journal of Operational Research, 150, 237-252.

Scholl, A. (2001). Robuste Planung und Optimierung. Heidelberg: Physica.

Selçuk, B., Fransoo, J. C., \& De Kok, A. G. (2008). Work-in-process clearing in supply chain operations planning. IIE Transactions, 40, 206-220. 
Sheikh, S., Komaki, M., \& Malakooti, B. (2015). Integrated risk and multiobjective optimization of energy systems. Computers 83 Industrial Engineering, 90, 1-11.

Söhner, V., \& Schneeweiss, C. (1995). Hierarchically integrated lot size optimization. European Journal of Operational Research, 86, 73-90.

van Arnum, P. (2000). Bulls or bears? Outlook in contract manufacturing. Chemical Market Reporter, 257, FR3-FR6.

Vandaele, N., van Nieuwenhuyse, I., \& Cupers, S. (2003). Optimal grouping for a nuclear magnetic resonance scanner by means of an open queueing model. European Journal of Operational Research, 151, 181-192.

Vandaele, N. J., \& Decouttere, C. J. (2013). Sustainable R\&D portfolio assessment. Decision Support Systems, 54, 1521-1532.

Vandaele, N. J., Lambrecht, M. R., Schuyter, N. d., \& Cremmery, R. (2000). Spicer Off-Highway Products Division-Brugge improves its lead-time and scheduling performance. Interfaces, 30, 83-95. 